Review began 12/01/2021 Review ended 12/14/2021 Published 12/23/2021

๑) Copyright 2021

Thiagarajan et al. This is an open access article distributed under the terms of the Creative Commons Attribution License CCBY 4.0., which permits unrestricted use, distribution, and reproduction in any medium, provided the original author and source are credited.

\section{Correlation Between Clinical Diagnosis, MRI, and Arthroscopy in Diagnosing Shoulder Pathology}

\author{
Aarthi Thiagarajan ${ }^{1}$, Raghu Nagaraj ${ }^{2}$, Kiran Marathe ${ }^{1}$ \\ 1. Orthopaedics and Trauma, Hosmat Hospital, Bangalore, IND 2. Orthopaedics and Trauma, Fortis Hospital, \\ Bangalore, IND \\ Corresponding author: Raghu Nagaraj,rgh_nagaraj@yahoo.com
}

\section{Abstract \\ Introduction}

Shoulder disorders are frequently encountered by clinicians and are a common cause of musculoskeletal pain in the general population. Clinical tests specific to each shoulder pathology, MRI, and arthroscopy are the most relied upon modalities of diagnosis used by many clinicians. The aim of this study was to correlate clinical tests and MRI with arthroscopy as the gold standard and whether a negative MRI with a positive clinical test could justify an arthroscopy.

\section{Materials and methods}

A total of 120 consecutive patients who had a history of shoulder pain or instability were evaluated by clinical tests and MRI, and underwent arthroscopy. Based on the confirmatory findings of arthroscopy, they were classified as True Positive (TP), True Negative (TN), False Positive (FP) and False Negative (FN) for each modality i.e., clinical tests and MRI.

\section{Results}

Clinical assessment of rotator cuff tears in comparison to arthroscopy yielded a sensitivity of $96.88 \%$, specificity of $92.86 \%$ and diagnostic accuracy of $95 \%$, whilst MRI had a sensitivity of $90.62 \%$, specificity of 92.86\% and diagnostic accuracy of $91.67 \%$. In anterior labral lesions, clinical assessment had a sensitivity of $94.44 \%$, specificity of $97.62 \%$ and diagnostic accuracy of $96.67 \%$, whilst MRI had a sensitivity of $83.33 \%$, specificity of $92.86 \%$, with diagnostic accuracy of $90 \%$. Interestingly, in the clinical assessment of superior labral tear from anterior to posterior (SLAP) lesions, a sensitivity of $90 \%$, specificity of $95 \%$, and diagnostic accuracy of $93.33 \%$ were observed while MRI had a sensitivity of $60 \%$, specificity of $92.50 \%$, and diagnostic accuracy of $81.67 \%$.

\section{Conclusion}

On the basis of these results, clinical assessment appears to be an effective tool in diagnosing shoulder pathologies, whereas MRI, though reliable in the identification of rotator cuff tears and instability, does not identify patients with SLAP lesions effectively. This study reinforces the importance of a good clinical examination of the shoulder, especially in chronic pain and an uncertain MRI, therefore improving patient management.

Categories: Orthopedics

Keywords: slap diagnosis, clinical assessment in shoulder, mri in shoulder, diagnostic modalities in shoulder, shoulder pathology

\section{Introduction}

Shoulder disorders are frequently encountered by clinicians and are the third most common cause of musculoskeletal pain in the general population [1]. Both generic and shoulder specific measurements have shown a significant impact on the health of the affected individual in domains like pain, distress, physical disability to name a few $[2,3]$. We cannot ignore the ramifications of the impact like monitory loss and the struggle to perform everyday activities, thus enhancing the requirement of accurate diagnosis and precise treatment.

Ideal clinical tests should be repeatable, easy to perform, sensitive, specific, and identify the origin of pathology in the shoulder joint. The accuracy of clinical tests varies according to clinicians as well as patients $[4,5]$. Moreover, the studies published validating these clinical tests are being questioned for their accuracy and clinical predictability $[6,7,8]$. Also, the heterogenicity of these studies poses a challenge for meta-analysis [9]. The paucity in the literature is hence a major concern since validation requires an easily accessible standard of reference. 


\section{Cureus}

MRI, being non-invasive and highly sensitive in diagnosing shoulder pathology, has a revelatory impact on the diagnosis [10]. Though contraindicated in patients with cardiac pacemakers and ferromagnetic implants, MRI is arguably the most widely used imaging modality in shoulder disorders.

Despite the evolution of MRI in detecting shoulder pathology and given the wide variability in the accuracy of MRI, several lesions are missed. In particular, the accuracy of MRI in the detection of partial rotator cuff tears and tendinitis is low and inconsistent in labral pathology in some centres with no dedicated musculoskeletal radiologists and higher device strength $[11,12]$.

The aim of this study was to correlate the various modalities of diagnosis of shoulder pathology with arthroscopy as the gold standard and to find out whether a negative MRI with a positive clinical test could justify an arthroscopy.

\section{Materials And Methods}

The population for this study consisted of 120 consecutive patients from a tertiary care orthopaedic centre with a history of shoulder pain or instability between September 2018 and August 2019. Ethical clearance was obtained for this study from the Hosmat Hospital Ethical Committee.

For the purpose of this study, the shoulder pathologies were organised into (a) Impingement, (b) rotator cuff tears - isolated or with other associations, (c) glenohumeral instability, and (d) glenoid labral lesions further classified as anterior labral (Bankart lesion and associated), superior labral (superior labral tear from anterior to posterior (SLAP) - isolated or associated with biceps tendon abnormalities), and posterior labral. As, osteochondral defects of the humerus and glenoid do not have a popular, validated clinical test, they were not included as an individual entity in the assessment. Osteoarthritis of the glenohumeral and acromioclavicular joint and acromioclavicular joint dislocation were excluded.

Clinical tests of the shoulder joint were performed by shoulder specialists. Hawkins test, modified Neer test, and painful arc test were used to evaluate impingement clinically. Positive apprehension and Jobe Relocation test helped to deduce instability. The tests for SLAP, due to their well-known enigma, were always used as a merger. Amongst the anterior slide test, active compression (O'Brien) test, Speed's test, biceps load tests, compression rotation test, and resisted supination external rotation test, a tailored combination of tests was selected by the shoulder specialists. Rotator cuff tears were identified with the empty can test, inability to abduct, external rotation lag sign, Hornblower's sign, belly press, and Gerber's lift-off test. MRI was performed in all patients who were symptomatic after failed conservative management and who were undergoing arthroscopy. A 1.5 T MRI was used and a single musculoskeletal radiologist reported all images. Images were obtained in the axial, coronal oblique, and sagittal oblique planes. The clinical and MRI diagnoses were categorised after the arthroscopy, as described in Table 1.
Categories
Description
True Positive (TP)
Clinical diagnosis/MRI diagnosis of a pathology, confirmed on arthroscopy
True Negative (TN)
Clinical diagnosis/MRI diagnosis of no pathology, confirmed on arthroscopy
False Positive (FP)
Clinical diagnosis/MRI diagnosis of a pathology, not confirmed on arthroscopy
False Negative (FN)
Clinical diagnosis/MRI diagnosis of no pathology, but pathology confirmed on arthroscopy.

TABLE 1: Categories based on clinical diagnosis/MRI diagnosis after arthroscopy

The sensitivity, specificity, positive predictive value (PPV), negative predictive value (NPV), likelihood of a positive test, likelihood of a negative test, and diagnostic accuracy of the clinical tests and MRI for each shoulder pathology was calculated with arthroscopic findings as the gold standard of reference. Data analysis were done using IBM SPSS Statistics for Windows, Version 26.0 (Released 2019, IBM Corp., Armonk, New York).

\section{Results}

The study population was 120 with a mean age of 41 years and a range of 20 to 79 years. There were 73 males and 47 females. In our study, the right side was more commonly affected than the left side. The mechanism of injury was degenerative in $38 \%$, sports-related in $35 \%$, and direct trauma in $27 \%$ of the study population.

\section{Rotator cuff tears}




\section{Cureus}

In our study, rotator cuff tears were identified arthroscopically in 64 patients, out of which 62 were identified by clinical assessment, showing a sensitivity of $96.88 \%$ and specificity of $92.86 \%$ and a diagnostic accuracy of $95 \%$ (Table 2).

\begin{tabular}{|c|c|c|}
\hline Parameter & Estimate & $95 \% \mathrm{Cl}$ \\
\hline Sensitivity & $96.88 \%$ & $83.78-99.92$ \\
\hline Specificity & $92.86 \%$ & $76.50-99.12$ \\
\hline Positive predictive value & $93.94 \%$ & $80.28-98.33$ \\
\hline Negative predictive value & $96.30 \%$ & $79.02-99.45$ \\
\hline Likelihood ratio of a positive test & 13.56 & $3.56-51.64$ \\
\hline Likelihood ratio of a negative test & 0.03 & $0.00-0.23$ \\
\hline Diagnostic accuracy & $95 \%$ & $86.08-98.96$ \\
\hline
\end{tabular}

TABLE 2: Clinical assessment of rotator cuff tears in comparison to arthroscopy

Out of 64 rotator cuff tears, 58 were identified by MRI, showing a sensitivity of $90.62 \%$ and specificity of $92.86 \%$ and a diagnostic accuracy of $91.67 \%$ (Table 3 ).

\begin{tabular}{|c|c|c|}
\hline Parameter & Estimate & $95 \% \mathrm{Cl}$ \\
\hline Sensitivity & $90.62 \%$ & $74.98-98.02$ \\
\hline Specificity & $92.86 \%$ & $76.50-99.12$ \\
\hline Positive predictive value & $93.55 \%$ & $79.15-98.23$ \\
\hline Negative predictive value & $89.66 \%$ & $74.60-96.24$ \\
\hline Likelihood ratio of a positive test & 12.69 & $3.32-48.46$ \\
\hline Likelihood ratio of a negative test & 0.10 & $0.03-0.30$ \\
\hline Diagnostic accuracy & $91.67 \%$ & $81.61-97.24$ \\
\hline
\end{tabular}

TABLE 3: MRI of rotator cuff tears in comparison to arthroscopy

\section{Bankart lesions}

Arthroscopy identified 36 Bankart lesions amongst the unstable shoulders. Of these, clinical assessment identified 34, showing a sensitivity of $94.44 \%$, specificity of $97.62 \%$, and diagnostic accuracy of $96.67 \%$ (Table 4). 


\section{Cureus}

\begin{tabular}{|c|c|c|}
\hline Parameter & Estimate & $95 \% \mathrm{Cl}$ \\
\hline Sensitivity & $94.44 \%$ & $72.71-99.86$ \\
\hline Specificity & $97.62 \%$ & 87.43- 99.94 \\
\hline Positive predictive value & $94.44 \%$ & $70.96-99.16$ \\
\hline Negative predictive value & $97.62 \%$ & 85.91- 99.64 \\
\hline Likelihood ratio of a positive test & 39.68 & $5.7-275.9$ \\
\hline Likelihood ratio of a negative test & 0.06 & $0.01-0.38$ \\
\hline Diagnostic accuracy & $96.67 \%$ & 88.47-99.59 \\
\hline
\end{tabular}

\section{TABLE 4: Clinical assessment of Bankart's lesion in comparison to arthroscopy}

MRI identified 30 Bankart lesions, showing a sensitivity of $83.33 \%$, specificity of $92.86 \%$, and diagnostic accuracy of $90 \%$ (Table 5).

\begin{tabular}{|c|c|c|}
\hline Parameter & Estimate & $95 \% \mathrm{Cl}$ \\
\hline Sensitivity & $83.33 \%$ & $58.58-96.42$ \\
\hline Specificity & $92.86 \%$ & $80.52-98.50$ \\
\hline Positive predictive value & $83.33 \%$ & $62.24-93.82$ \\
\hline Negative predictive value & $92.86 \%$ & $82.18-97.34$ \\
\hline Likelihood ratio of a positive test & 11.67 & $3.85-35.40$ \\
\hline Likelihood ratio of a negative test & 0.18 & $0.06-0.51$ \\
\hline Diagnostic accuracy & $90 \%$ & $79.49-96.24$ \\
\hline
\end{tabular}

TABLE 5: MRI of Bankart's lesion in comparison to arthroscopy

\section{SLAP tears}

Superior labral tears were identified in 40 patients arthroscopically, out of which 36 were identified through clinical assessment showing a sensitivity of $90 \%$, specificity of $95 \%$, and diagnostic accuracy of $93.33 \%$ (Table 6).

\begin{tabular}{|l|ll}
\hline Parameter & Estimate & $\mathbf{9 5 \%} \mathbf{C l}$ \\
\hline Sensitivity & $90 \%$ & $68.30-98.77$ \\
\hline Specificity & $95 \%$ & $83.08-99.39$ \\
\hline Positive predictive value & $90 \%$ & $69.82-97.22$ \\
\hline Negative predictive value & $95 \%$ & $83.59-98.61$ \\
\hline Likelihood ratio of a positive test & 18 & $4.63-70.04$ \\
\hline Likelihood ratio of a negative test & 0.11 & $0.03-0.39$ \\
\hline Diagnostic accuracy & $93.33 \%$ & $83.80-98.15$
\end{tabular}

TABLE 6: Clinical assessment of SLAP tears in comparison to arthroscopy 


\section{Cureus}

MRI could identify only 24 out of the 40 SLAP tears identified by arthroscopy, showing a sensitivity of $60 \%$, specificity of $92.50 \%$, and diagnostic accuracy of $81.67 \%$ (Table 7 ).

\begin{tabular}{|c|c|c|}
\hline Parameter & Estimate & $95 \% \mathrm{Cl}$ \\
\hline Sensitivity & $60 \%$ & $36.05-80.88$ \\
\hline Specificity & $92.50 \%$ & $79.61-98.43$ \\
\hline Positive predictive value & $80 \%$ & $55.99-92.64$ \\
\hline Negative predictive value & $82.22 \%$ & $72.86-88.85$ \\
\hline Likelihood ratio of a Positive Test & 8 & $2.54-25.16$ \\
\hline Likelihood ratio of a Negative Test & 0.43 & $0.25-0.75$ \\
\hline Diagnostic accuracy & $81.67 \%$ & $69.56-90.84$ \\
\hline
\end{tabular}

TABLE 7: MRI of SLAP tears in comparison to arthroscopy

\section{Discussion}

A clinician's ability to diagnose different shoulder pathologies based on his assessment alone has been questioned by several studies. In instances where either the clinics are busy or the pathology is acute and painful, a thorough clinical assessment is forsaken, and as a matter of course radiological investigations like MRI, magnetic resonance angiogram (MRA) are banked upon. Meta-analysis of three fairly popular clinical tests - Modified Neer test, Hawkins-Kennedy test, and Speed's test, used to examine impingement and SLAP lesion, concluded that none of these tests is diagnostic for their stated pathology [9]. Thus comes the very important question of selecting the diagnostic modality for accurate diagnosis of shoulder pathology and decision-making on surgical intervention.

\section{Rotator cuff tears}

In our study, rotator cuff tears were identified arthroscopically in 64 patients. Clinical assessment diagnosed rotator cuff tears in 62 patients, whereas MRI identified 58 out of 64 patients.

Dinnes et al. [13] in their study showed that clinical examination in diagnosing rotator cuff tears had sensitivity of $90 \%$ but specificity of $54 \%$, while MRI in diagnosing rotator cuff tears had sensitivity of $89 \%$ and specificity of $93 \%$, which is comparable to our study. Another study by Torensten et al. [12] showed identification of rotator cuff tears by MRI with a sensitivity of $96 \%$. Fukuda et al. [14] described that there is a $7.2 \%$ incidence of purely intra-tendinous supraspinatus tear. This is paramount in deducing the accuracy of MRI, which identifies the presence of intra- substance rotator cuff tears but are missed arthroscopically, thus falsely reducing the accuracy of MRI if the tears do not communicate with either the subacromial space or glenohumeral joint. Vice versa, surgical interventions based on intrasubstance tears would not be beneficial for the patient. MRI unarguably identified rotator cuff tears effectively in our study; however, its inability to clearly differentiate partial and complete tears places a surgeon, who is relying on MRI, in a crux to take a surgical decision upon intervention.

\section{Bankart tears}

Amongst the clinically unstable shoulders, Bankart lesion was found arthroscopically in 36 patients. Clinical examination picked up the pathology in 34 patients and MRI picked it up in 30. In their study, Loh et al. [15] showed that clinical examination diagnosed Bankart lesion with a sensitivity of $94 \%$ while MRI had $89 \%$ sensitivity, which was comparable to our study. Although Green et al. [16] concluded in their study that MRI is not useful in anterior shoulder instability, we had a very high diagnostic accuracy in our study.

\section{SLAP tears}

A total of 40 patients were known to have SLAP tears through arthroscopy. Clinical examination helped to pick 36 of them, while MRI identified only 24. In their study, Liu et al. [17] recognised that clinical examination yielded a sensitivity of $90 \%$ and specificity of $85 \%$, and MRI yielded a sensitivity of $59 \%$ and specificity of $85 \%$, which is comparable to our study. Unlike the evaluation of other shoulder disorders, identification of SLAP lesions by MRI was poor owing to reduced sensitivity and diagnostic accuracy. Nevertheless, studies do show that provocative manoeuvres like longitudinal traction of the arm or positioning the shoulder in abduction and external rotation during imaging or additional planes parallel to the biceps tendon improve the diagnostic accuracy of MRI in SLAP tears $[18,19,20]$. 
Several studies have identified the sensitivity of MRA in detecting glenoid labral lesions, but we need to bear in mind the financial burden on the patient and the rarer complications like contrast allergy, extravasation of contrast, and prolonged waiting time for the investigation thus delaying the definite management of the patient. Moreover, a majority of studies recommending MRA identified the patient group at the time of arthroscopy, retrospectively evaluating those with proven labral lesions and so, questioning the results. Thus, the selection of operative candidates or assistance in planning the surgical procedure in SLAP lesions would demand a tailormade clinical assessment of a symptomatic patient. We cannot ignore the fact that clinical tests in our study were done by shoulder specialists and their knowledge and experience could have improved our results in comparison to studies where clinical assessments were performed by non-specialist orthopaedic surgeons.

\section{Conclusions}

On the basis of these results, clinical assessment appears to be an effective tool in diagnosing shoulder pathologies, whereas MRI, though reliable in the identification of rotator cuff tears and instability, does not identify patients with SLAP lesions effectively. We recommend going ahead with surgical treatment of patients who have been clinically identified to have a slap tear, in spite of a negative MRI, to provide better results after a sufficient period of failed conservative treatment. Considering that clinical examination has shown higher sensitivity and specificity in all the shoulder conditions, a good clinical examination will not miss pathology in most patients. This study reinforces the importance of a good clinical examination of the shoulder, especially in chronic pain and an uncertain MRI, and warrants the need for an arthroscopy, therefore improving patient management.

\section{Additional Information \\ Disclosures}

Human subjects: Consent was obtained or waived by all participants in this study. Hosmat Hospital Ethical Committee issued approval Dated June 5, 2019. This is to certify that the study titled "Correlation between clinical diagnosis, MRI, and Arthroscopy in diagnosing shoulder pathology" has been reviewed, discussed, and approved by the board of members of the ethical committee. . Animal subjects: All authors have confirmed that this study did not involve animal subjects or tissue. Conflicts of interest: In compliance with the ICMJE uniform disclosure form, all authors declare the following: Payment/services info: All authors have declared that no financial support was received from any organization for the submitted work. Financial relationships: All authors have declared that they have no financial relationships at present or within the previous three years with any organizations that might have an interest in the submitted work. Other relationships: All authors have declared that there are no other relationships or activities that could appear to have influenced the submitted work.

\section{References}

1. Urwin M, Symmons D, Allison T, et al.: Estimating the burden of musculoskeletal disorders in the community: the comparative prevalence of symptoms at different anatomical sites, and the relation to social deprivation. Ann Rheum Dis. 1998, 57:649-55. 10.1136/ard.57.11.649

2. Ostör AJ, Richards CA, Prevost AT, Speed CA, Hazleman BL: Diagnosis and relation to general health of shoulder disorders presenting to primary care. Rheumatology (Oxford). 2005, 44:800-5. 10.1093/rheumatology/keh598

3. Hill CL, Gill TK, Shanahan EM, Taylor AW: Prevalence and correlates of shoulder pain and stiffness in a population-based study: the North West Adelaide Health Study. Int J Rheum Dis. 2010, 13:215-22. 10.1111/j.1756-185X.2010.01475.x

4. Magee DJ: The shoulder. Orthopaedic Physical Assessment. W. B. Saunders Company, Philadelphia; 1992. 2nd edition:90-142.

5. Palmer K, Walker-Bone K, Linaker C, Reading I, Kellingray S, Coggon D, Cooper C: The Southampton examination schedule for the diagnosis of musculoskeletal disorders of the upper limb. Ann Rheum Dis. 2000, 59:5-11. 10.1136/ard.59.1.5

6. Deeks JJ: Systematic reviews in health care: systematic reviews of evaluations of diagnostic and screening tests. BMJ. 2001, 323:157-62. 10.1136/bmj.323.7305.157

7. Knottnerus JA, van Weel C, Muris JW: Evaluation of diagnostic procedures . BMJ. 2002, 324:477-80. 10.1136/bmj.324.7335.477

8. Reid MC, Lachs MS, Feinstein AR: Use of methodological standards in diagnostic test research. Getting better but still not good. JAMA. 1995, 274:645-51.

9. Hegedus EJ, Goode A, Campbell S, Morin A, Tamaddoni M, Moorman CT 3rd, Cook C: Physical examination tests of the shoulder: a systematic review with meta-analysis of individual tests. Br J Sports Med. 2008, 42:80-92. 10.1136/bjsm.2007.038406

10. Blanchard TK, Mackenzie R, Bearcroft PW, et al.: Magnetic resonance imaging of the shoulder: assessment of effectiveness. Clin Radiol. 1997, 52:363-8. 10.1016/s0009-9260(97)80131-6

11. Iannotti JP, Zlatkin MB, Esterhai JL, Kressel HY, Dalinka MK, Spindler KP: Magnetic resonance imaging of the shoulder. Sensitivity, specificity, and predictive value. J Bone Joint Surg Am. 1991, 73:17-29.

12. Torstensen ET, Hollinshead RM: Comparison of magnetic resonance imaging and arthroscopy in the evaluation of shoulder pathology. J Shoulder Elbow Surg. 1999, 8:42-5. 10.1016/s1058-2746(99)90053-8

13. Dinnes J, Loveman E, McIntyre L, Waugh N: The effectiveness of diagnostic tests for the assessment of shoulder pain due to soft tissue disorders: a systematic review. Health Technol Assess. 2003, 7:iii, 1-166. 


\section{Cureus}

10.3310/hta7290

14. Fukuda H, Hamada K, Nakajima T, Tomonaga A: Pathology and pathogenesis of the intratendinous tearing of the rotator cuff viewed from en bloc histologic sections. Clin Orthop Relat Res. 1994, 60-7.

15. Loh B, Lim JB, Tan AH: Is clinical evaluation alone sufficient for the diagnosis of a Bankart lesion without the use of magnetic resonance imaging?. Ann Transl Med. 2016, 4:419. 10.21037/atm.2016.11.22

16. Green MR, Christensen KP: Magnetic resonance imaging of the glenoid labrum in anterior shoulder instability. Am J Sports Med. 1994, 22:493-8. 10.1177/036354659402200410

17. Liu SH, Henry MH, Nuccion S, Shapiro MS, Dorey F: Diagnosis of glenoid labral tears: a comparison between magnetic resonance imaging and clinical examinations. Am J Sports Med. 1996, 24:149-54. $10.1177 / 036354659602400205$

18. Chan KK, Muldoon KA, Yeh L, et al.: Superior labral anteroposterior lesions: MR arthrography with arm traction. AJR Am J Roentgenol. 1999, 173:1117-22. 10.2214/ajr.173.4.10511190

19. Chhadia AM, Goldberg BA, Hutchinson MR: Abnormal translation in SLAP lesions on magnetic resonance imaging abducted externally rotated view. Arthroscopy. 2010, 26:19-25. 10.1016/j.arthro.2009.06.028

20. Borrero CG, Casagranda BU, Towers JD, Bradley JP: Magnetic resonance appearance of posterosuperior labral peel back during humeral abduction and external rotation. Skeletal Radiol. 2010, 39:19-26. 10.1007/s00256-009-0744-4 